\title{
Blood culture negative endocarditis in the modern era of 16S rRNA sequencing
}

\author{
Authors: Rebecca Godfrey, ${ }^{A}$ Sally Curtis, ${ }^{B}$ William HK Schilling ${ }^{C}$ and P Rachael James ${ }^{D}$
}

\begin{abstract}
Blood culture negative endocarditis (BCNE) accounts for up to $20 \%$ of infective endocarditis. While the most common cause of $\mathrm{BCNE}$ remains the initiation of antibiotics prior to culture, intracellular organisms such as Coxiella and Bartonella spp account for a significant proportion of cases. Identifying the infecting organism remains important to ensure optimal antimicrobial treatment. However, these organisms can be difficult to diagnose. We outline a systematic approach to BCNE. Over half of patients with infective endocarditis now undergo early surgery and $16 \mathrm{~S}$ ribosomal ribonucleic acid (rRNA) polymerase chain reaction (PCR) of excised tissue can be vitally important to secure a diagnosis. Molecular testing is likely to become a key tool in improving outcomes from BCNE and contribute to an improved understanding of the aetiology. We advocate modifying the Duke criteria to incorporate organisms identified on molecular testing, including 16S rRNA $\mathrm{PCR}$, in particular from explanted tissue.
\end{abstract}

KEYWORDS: Endocarditis, culture negative, 16S rRNA, PCR, Bartonella henselae

DOI: $10.7861 /$ clinmed.2019-0342

\section{Case presentation}

A 34-year-old man who worked as a delivery driver and was usually fit and well, was admitted with a two-week history of breathlessness on exertion and a dry cough. He was afebrile, had signs of severe aortic regurgitation and pulmonary congestion. There were no peripheral stigmata of endocarditis. Blood tests showed white blood cells $7.2 \times 10^{9} / \mathrm{L}$, C-reactive protein $33 \mathrm{mg} / \mathrm{L}$, haemoglobin $92 \mathrm{~g} / \mathrm{L}$ and creatinine $151 \mu \mathrm{mol} / \mathrm{L}$. He was started on diuretics and transthoracic echocardiography demonstrated a bicuspid aortic valve with severe aortic regurgitation and several vegetations up to $1.8 \mathrm{~cm}$ in length. The patient underwent an urgent aortic valve replacement on haemodynamic grounds.

Several sets of peripheral blood cultures were negative. There was no history of antibiotic administration in the community.

Authors: ${ }^{\text {A }}$ cardiology fellow, Sussex Cardiac Centre, Brighton, UK; ${ }^{B}$ microbiology consultant, Brighton and Sussex University Hospitals

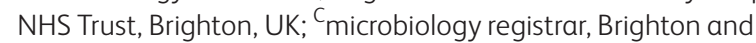
Sussex University Hospitals NHS Trust, Brighton, UK; ${ }^{\text {D }}$ consultant cardiologist, Sussex Cardiac Centre, Brighton, UK
The patient was treated with high dose intravenous amoxicillin and gentamicin and clinically improved. Coxiella burnetii serology was negative and the patient was negative for HIV infection. Currently there are no serological tests for Bartonella spp in the UK. A diagnosis of blood culture negative endocarditis was made and the excised valve tissue was referred for molecular sequencing. $16 \mathrm{~S}$ ribosomal ribonucleic acid (rRNA) bacterial polymerase chain reaction (PCR) analysis was positive for Bartonella henselae, the causative pathogen of cat scratch disease. The antibiotic regimen was changed to doxycycline $100 \mathrm{mg}$ twice daily and gentamicin. This regimen has increased efficacy in treating Bartonella spp endocarditis and is advised by the European Society of Cardiology guidelines. ${ }^{1}$ The patient was discharged on oral doxycycline to complete treatment and made a full recovery. We discovered that the patient's partner worked for a cat rescue centre and occasionally they would care for abandoned cats at home.

Bartonella spp are a recognised cause of blood culture negative endocarditis (BCNE). ${ }^{2,3}$ Although six Bartonella spp are known to cause infective endocarditis in humans, Bartonella hensalae and Bartonella quintana (transmitted by human body lice and historically called 'trench fever', now increasingly found in the homeless population) account for $95 \%$ of cases..$^{4-6}$ Bartonella is increasingly being recognised as a cause of endocarditis in the UK? Most patients have pre-existing valvular disease as in this case and infection with Bartonella spp typically results in destructive endocarditis with a high incidence of valve replacement, as high as $80 \%$ in one series. $3,4,6,8$ This case highlights the importance of history taking even in the modern era as being scratched by a cat, or coming into contact with cat fleas are important risk factors for Bartonella hensalae infection. ${ }^{3,4,8-10}$ The serodiagnostic service for Bartonella was withdrawn in the UK in 2015 following a review by Public Health England (personal communication with Dr Colin Brown, Reference Lab 2019). At least one large centre sends blood samples for serology to a French research laboratory, as they see a relatively large number of cases of Bartonella spp in the homeless population. The contemporary diagnosis of Bartonella endocarditis in the UK is made predominantly through molecular sequencing of infected tissue or from culture. The latter being lengthy, difficult and thus rarely performed. Bartonella specific PCR has a high sensitivity from valve tissue of up to $92 \% .^{11}$

\section{Introduction}

Despite advances in medicine endocarditis continues to be associated with significant morbidity and mortality. Timely diagnosis for a condition with protean presentation remains 
challenging. Delayed diagnosis prevents active management with optimal antimicrobial therapy, early involvement from a multidisciplinary endocarditis team and individualised patient management including early surgery which have all been shown to significantly improve outcomes. ${ }^{12}$

Most cases of endocarditis are caused by bacterial infection and usually the diagnosis is made by culture-dependent methods. Uncertainty about the causative organism may result in inadequate treatment, exposure of the patient to potentially toxic empirical treatment and ultimately affect outcome. A systematic approach to BCNE is needed.

Modern practice, using conventional automated blood culture systems and conventional media subculture for the standard duration (7 days in endocarditis), will yield organisms historically perceived as fastidious or slow-growing including the HACEK (Haemophilus, Aggregatibacter, Cardiobacterium, Eikenella, Kingella) group organisms, nutritionally variant Streptococci and Candida species. ${ }^{13,14}$

\section{Prevalence and aetiology}

Despite there being a near constant bacteraemia before initiation of appropriate antibiotics, approximately $12-20 \%$ patients remain culture negative with no growth after 7 days incubation. ${ }^{15,16}$ The prevalence of $\mathrm{BCNE}$ varies in the literature, largely reflecting the populations investigated and in particular, whether cases where prior antibiotics were administered are included.

It is widely recognised that the most common cause of BCNE is the initiation of antibiotics prior to culture. In one UK series, looking specifically at patients with BCNE found, ultimately, to have Gram-positive cocci causing infection, two-thirds had received antibiotics prior to culture. ${ }^{16}$

True BCNE is caused by infection with a variety of bacteria including Coxiella burnetii (Q fever), Bartonella spp, Brucella spp, Tropheryma whipplei, Mycobacteria species and non-candidal fungi (Table 1). These bacteria are either intracellular organisms, or are unlikely to grow on conventional media. Many of the true BCNE organisms are associated with specific risk factors or exposures and careful history taking is of paramount importance (Table 2). Non-infective thrombotic endocarditis is rare, accounting for $2.2 \%$ of BCNE in one French study from a BCNE reference centre and is associated with certain cancers and autoimmune diseases such as systemic lupus erythematosus (Table 2). ${ }^{17}$

Identification of the causative organism in BCNE allows refinement of empirical antibiotic regimens. Standard empirical treatment for BCNE risks exposure of the patient to unnecessary toxicity and sub-optimal antimicrobial treatment.

\section{Presentation}

Evidence suggests that culture positive and culture negative endocarditis present similarly although heart failure is more prevalent (likely reflecting the delay in diagnosis) and overt sepsis less common in BCNE as in the case presented. ${ }^{17,22,23}$ Echocardiography is less likely to be diagnostic in some BCNE infections eg cases of Coxiella burnetii endocarditis, where vegetations are often small. ${ }^{22,24,25}$

\section{Identifying the causative organism in BCNE}

We suggest the following pragmatic approach to determining the causative organism, in line with current guidelines and
Table 1. Causes of blood culture negative

endocarditis

\begin{tabular}{|c|c|c|}
\hline \multirow{9}{*}{$\begin{array}{l}\text { Antibiotics given } \\
\text { prior to taking } \\
\text { blood cultures }\end{array}$} & True BCNE & $\begin{array}{l}\text { Non-infective } \\
\text { BCNE }\end{array}$ \\
\hline & Coxiella & Behçet's \\
\hline & Bartonella spp & disease \\
\hline & Brucella & Systemic lupis \\
\hline & Tropheryma whipplei & erythematosus \\
\hline & Mycobacteria spp (M & endocarditis) \\
\hline & $\begin{array}{l}\text { chimera following cardiac } \\
\text { surgery) }\end{array}$ & $\begin{array}{l}\text { Marantic } \\
\text { (breast/lung/ }\end{array}$ \\
\hline & Fungi (Aspergillus) & prostate/colon \\
\hline & $\begin{array}{l}\text { May be diagnosed by: } \\
\text { > Serology (Bartonella } \\
\text { currently unavailable in } \\
\text { UK) } \\
>\text { PCR of excised valve / } \\
\text { embolectomy material }\end{array}$ & cancer) \\
\hline
\end{tabular}

$\mathrm{BCNE}=$ blood culture negative endocarditis.

evidence (Fig 1). History taking may identify recognised host and epidemiological factors associated with different aetiologies of BCNE (Table 2). Determining these factors, including immune status eg HIV status informs further diagnostic testing.

\section{Serology}

Serology for Coxiella burnetii is first line in the investigation of BCNE; Coxiella phase 1 immunoglobulin $\mathrm{G}(\mathrm{IgG}) \geq 800$ is diagnostic of Coxiella burnetii infection and qualifies as a major Duke criterion. ${ }^{27}$ Although Bartonella spp IgG $\geq 800$ is diagnostic for Bartonella spp infection, serological testing as stated is not currently available in the UK. ${ }^{28}$ In countries where serology is available, it should be recognised that cross-reactivity occurs between Bartonella spp and Coxiella burnetii and it is notoriously difficult to interpret serology in this setting. ${ }^{14}$

The British Society for Antimicrobial Chemotherapy (BSAC) advise considering Brucella serology in the setting of dietary or travel-related exposure (Table 2). ${ }^{26}$ Mycoplasma, Legionella and Mycobacteria endocarditis are exceptionally rare. ${ }^{17,29}$ Routine serological testing for Legionella, Mycoplasma and Chlamydia spp is not recommended. ${ }^{26}$ Previous serological Chlamydia spp diagnoses may reflect cross-reactivity with Bartonella spp (with no valve positives to date). ${ }^{29,30}$ In two large studies evaluating a range of serological tests in $\mathrm{BCNE}, 624$ patients were diagnosed by serology (from a total of 1,093), and only four cases BCNE would have been missed if serology testing had been restricted to testing for Coxiella burnetii and Bartonella spp only. ${ }^{15,17,31}$

\section{Examination of explanted tissue}

Explanted valve tissue or embolectomy material is routinely sent for microscopy and culture, in part to guide duration of postoperative therapy (with a longer duration of postoperative antibiotic therapy recommended if the organism is grown from the valve). ${ }^{32}$ 
Table 2. Pathogens causing blood culture negative endocarditis

\begin{tabular}{|c|c|}
\hline Organism & Risk factors for infection \\
\hline $\begin{array}{l}\text { Coxiella burnetii (intracellular) } \\
28-37 \% \text { of } \mathrm{BCNE}^{15,17}\end{array}$ & $\begin{array}{l}\text { Occupational exposure to farm animals (sheep, cattle, goats) } \\
\text { Living downwind from farms and infected farm material (straw manure) } \\
\text { Abattoir workers } \\
\text { Laboratory exposure to pathogens } \\
\text { Immunosuppression possibly including HIV }{ }^{19}\end{array}$ \\
\hline Bartonella henselae (intracellular) & Contact with cats (higher bacteraemia in kittens) 20 \\
\hline $\begin{array}{l}\text { Bartonella quintana (intracellular) } \\
\text { Bartonella spp collectively } 12-28 \% \text { of } \mathrm{BCNE}^{15,17}\end{array}$ & $\begin{array}{l}\text { Contact with human body lice / homeless shelters } \\
\text { Chronic alcoholism } \\
\text { Travel to north Africa }\end{array}$ \\
\hline $\begin{array}{l}\text { Brucella (fastidious Gram-negative bacilli) } \\
\text { Only in endemic areas }\end{array}$ & $\begin{array}{l}\text { Occupational exposure to farm animals (sheep, cattle, goats) } \\
\text { Ingesting unpasteurised milk/cheese / undercooked meat } \\
\text { Travel to Middle East }\end{array}$ \\
\hline $\begin{array}{l}\text { Fungi } \\
1-2 \% \text { of } \mathrm{BCNE}^{15,17}\end{array}$ & $\begin{array}{l}\text { HIV positive } \\
\text { Indwelling venous catheter } \\
\text { Prior cardiac surgery }\end{array}$ \\
\hline $\begin{array}{l}\text { Tropheryma whipplei } \\
\text { Up to } 6 \% \text { of } \mathrm{BCNE}^{15,17,21}\end{array}$ & Occupational exposure to soil / farm animals \\
\hline Tuberculosis and other mycobacteria & $\begin{array}{l}\text { Tuberculosis contact or exposure } \\
\text { Mycobacterium chimera following cardiac surgery }\end{array}$ \\
\hline
\end{tabular}

Histopathological examination of the valve can be valuable. Specific culture media or histopathological stains can be used to look for Mycobacteria spp (alcohol and acid-fast bacilli), Tropheryma whipplei (periodic acid-Schiff positive macrophages), fungi (hyphae / silver stain) and Bartonella spp (Warthin-Starry stain). ${ }^{14}$

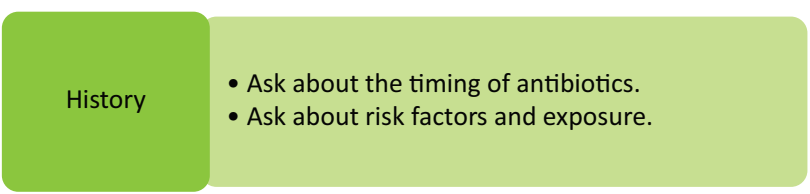

- Optimally filled.
- Septic: two separate sets within 1 hour. Subacute/
chronic: three sets each 6 hours apart.
- Alert microbiology laboratory to suspicion of
endocarditis, to extend culture to 7 days.

$\begin{array}{cl} & \text { - Serology: Coxiella burnetti, Bartonella spp } \\ \text { (currently unavailable in the UK). } \\ \text { If blood cultures } \\ \text { - Serology: Brucella if Coxiella negative and exposure. } \\ \text { are negative } & \text { - ESC/BSAC recommend considering Legionella, } \\ & \text { Mycoplasma, chlamydia serology. } 1,26 \\ \text { - ANA, RF, anti-dsDNA. }\end{array}$

Excised tissue (heart valve /

tissue from embolectomy)

- Tissue culture.

- Consider histopathology.

- Broad range 165 and 18 S rRNA PCR

Fig 1. A pragmatic approach to determining the causative organism in suspected endocarditis. ANA = anti-nuclear antibody; anti-dsDNA = antidouble-stranded deoxyribonucleic acid; BSAC = British Society for Antimicrobial Chemotherapy; ESC = European Society of Cardiology; $\mathrm{PCR}=$ polymerase chain reaction; $\mathrm{RF}$ rheumatoid factor; $r \mathrm{RNA}=$ ribosomal ribonucleic acid.

\section{Molecular}

Serum testing with PCR is not recommended due to low sensitivity and no additional cases of infection were diagnosed following initial serology in a large study. ${ }^{17}$

Molecular techniques to diagnose endocarditis from explanted tissue have been available for over 20 years and are becoming increasingly important in the diagnostic workup of endocarditis. ${ }^{30,33}$ Currently they remain excluded from the modified Duke criteria. One molecular technique, the pan-bacterial 16S rRNA PCR, uses PCR on homogenised tissue to amplify bacterial genetic material from the $16 \mathrm{~S}$ rRNA. This genetic material is conserved in all bacteria and hypervariable segments can allow for further identification of bacteria down to a species level. The technique is highly sensitive and specific for identifying the causative organism although clinical correlation is required and it is important to avoid cross-contamination of tissue. Microbial deoxyribonucleic acid can persist for months following infection and the presence of bacteria from PCR analysis does not necessarily imply ongoing infection.

The technique detects the causative organism in the majority of BCNE cases and represents a major advance in the management of cases of endocarditis where antibiotics were given prior to culture, in patients with equivocal serological results, in cases where culture and serology have been negative or, as in the case illustrated, where serological testing is unavailable. ${ }^{17,33}$ Furthermore, and importantly, molecular sequencing offers improved understanding of the true aetiology of endocarditis in different countries and is a major advance in the diagnosis and management of this devastating disease. ${ }^{34,35}$ Modification of the Duke criteria to include tissue PCR has been acknowledged in the latest update from the British Society for Antimicrobial Chemotherapy, however, this has not been formalised. ${ }^{26,36}$ Current international guidelines on endocarditis reference Duke criteria, excluding molecular testing. ${ }^{1}$ 


\section{Take home messages}

Endocarditis remains challenging to diagnose and manage as it is associated with a wide diversity of presentation and variable clinical course. The incidence of endocarditis is increasing, particularly in the elderly. All clinicians should have a low threshold to suspect the diagnosis in the setting of unexplained sepsis, a murmur and/or evidence of systemic embolisation particularly in those at increased risk such as native valve disease, prosthetic valve replacement or the presence of an intracardiac device.

The case highlights the need to act quickly when the patient is acutely unwell. Recognition of severe aortic regurgitation prompted early discussion with the endocarditis team and urgent cardiac surgery. Over recent years, there has been increasing recognition of the importance of an experienced endocarditis team perspective on tailored medical, surgical and antimicrobial management. Patients should be discussed urgently if there is haemodynamic instability, heart failure, recurrent systemic embolisation or uncontrolled infection.

A significant proportion of endocarditis is blood culture negative. This review highlights a pragmatic approach to these patients. In the vignette, BCNE was caused by Bartonella henselae infection and diagnosis of the causative organism was delayed by the current absence of serological testing in the UK. Although with hindsight there were epidemiological clues when an extended history was taken, 16S rRNA PCR analysis of tissue from the explanted valve was ultimately diagnostic and changed antimicrobial management.

$>$ Consider endocarditis early.

> Management of endocarditis with a multidisciplinary endocarditis team improves outcome through individualised care and early surgery.

> Blood culture negative endocarditis is common. In patients who undergo surgery, excised tissue should be sent for $16 \mathrm{~S}$ rRNA PCR. This can diagnose the causative organism and will inform optimal antimicrobial treatment and duration. This is of increasing relevance given that early surgical intervention is associated with a lower risk of mortality in infective endocarditis, and over $55 \%$ of patients undergo surgery early in their clinical course. ${ }^{37,38}$

> We advocate modifying the Duke criteria to include organisms detected on explanted tissue using molecular testing. This valuable test is likely to become a key tool in improving outcomes from BCNE and better understanding the aetiology.

\section{Acknowledgements}

The authors thank the patient for taking the time to discuss the presentation and management of his illness, and for his consent to submit this report for publication.

\section{References}

1 Habib G, Lancellotti P, Antunes MJ et al. 2015 ESC Guidelines for the management of infective endocarditis The Task Force for the Management of Infective Endocarditis of the European Society of Cardiology (ESC). Endorsed by: European Association for CardioThoracic Surgery (EACTS), the European Association of Nuclear Medicine (EANM). Eur Heart J 2015;36:3075-128.

2 Breathnach AS, Hoare JM, Eykyn SJ. Culture-negative endocarditis: contribution of bartonella infections. Heart 1997;77:474-6.
3 Raoult D, Fournier PE, Vandenesch F et al. Outcome and treatment of Bartonella endocarditis. Arch Intern Med 2003;163:226-30.

4 Raoult D, Fournier PE, Drancourt M et al. Diagnosis of 22 new cases of Bartonella endocarditis. Ann Intern Med 1996;125: 646-52.

5 Avidor B, Graidy M, Efrat G et al. Bartonella koehlerae, a new catassociated agent of culture-negative human endocarditis. J Clin Microbiol 2004:42:3462-8.

6 Raoult D, Roblot F, Rolain JM et al. First isolation of Bartonella alsatica from a valve of a patient with endocarditis. J Clin Microbiol 2006:44:278-9.

7 Chaloner GL, Harrison TG, Birtles RJ. Bartonella species as a cause of infective endocarditis in the UK. Epidemiol Infect 2013;141:841-6.

8 Fournier PE, Lelievre H, Eykyn S] et al. Epidemiologic and clinical characteristics of Bartonella quintana and Bartonella henselae endocarditis: a study of 48 patients. Medicine (Baltimore) 2001:80:245-51.

9 Spach DH, Kanter AS, Daniels NA et al. Bartonella (Rochalimaea) species as a cause of apparent "culture-negative" endocarditis. Clin Infect Dis 1995;20:1044-7.

10 Spach DH, Callis KP, Paauw DS et al. Endocarditis caused by Rochalimaea quintana in a patient infected with human immunodeficiency virus. J Clin Microbiol 1993;31:692-4.

11 Edouard S, Nabet C, Lepidi $\mathrm{H}$ et al. Bartonella, a common cause of endocarditis: a report on 106 cases and review. J Clin Microbiol 2015;53:824-9.

12 Davierwala PM, Marin-Cuartas M, Misfeld M et al. The value of an "Endocarditis Team". Ann Cardiothorac Surg 2019;8:621-9.

13 Murdoch DR, Corey GR, Hoen B et al. Clinical presentation, etiology, and outcome of infective endocarditis in the 21st century: the International Collaboration on Endocarditis-Prospective Cohort Study. Arch Intern Med 2009;169:463-73.

14 Liesman RM, Pritt BS, Maleszewski J] et al. Laboratory Diagnosis of Infective Endocarditis. J Clin Microbiol 2017;55:2599-608.

15 Houpikian P, Raoult D. Blood culture-negative endocarditis in a reference center: etiologic diagnosis of 348 cases. Medicine (Baltimore) 2005;84:162-73.

16 Lamas CC, Eykyn SJ. Blood culture negative endocarditis: analysis of 63 cases presenting over 25 years. Heart 2003;89:258-62.

17 Fournier PE, Thuny F, Richet $\mathrm{H}$ et al. Comprehensive diagnostic strategy for blood culture-negative endocarditis: a prospective study of 819 new cases. Clin Infect Dis 2010;51:131-40.

18 de Rooij MM, Borlee F, Smit LA et al Detection of Coxiella burnetii in ambient air after a large $Q$ fever outbreak. PLoS One 2016;11:e0151281.

19 Stein A, Raoult D. Q fever endocarditis. Eur Heart J 1995;16 Suppl B:19-23.

20 Fleischman DA, Chomel BB, Kasten RW et al. Bartonella Infection among Cats Adopted from a San Francisco Shelter, Revisited. Appl Environ Microbiol 2015;81:6446-50.

21 Geissdorfer W, Moos V, Moter A et al. High frequency of Tropheryma whipplei in culture-negative endocarditis. J Clin Microbiol 2012;50:216-22.

22 Ferrera C, Vilacosta I, Fernandez C et al. Reassessment of blood culture-negative endocarditis: its profile is similar to that of blood culture-positive endocarditis. Rev Esp Cardiol (Engl Ed) 2012;65:891-900.

23 Lamas CC, Fournier PE, Zappa M et al. Diagnosis of blood culture-negative endocarditis and clinical comparison between blood culture-negative and blood culture-positive cases. Infection 2016:44:459-66.

24 Maurin M, Raoult D. Q fever. Clin Microbiol Rev 1999;12:518-53.

25 Houpikian P, Habib G, Mesana T et al. Changing clinical presentation of Q fever endocarditis. Clin Infect Dis 2002;34:E28-31.

26 Gould FK, Denning DW, Elliott TS et al. Guidelines for the diagnosis and antibiotic treatment of endocarditis in adults: a report 
of the Working Party of the British Society for Antimicrobial Chemotherapy. J Antimicrob Chemother 2012;67:269-89.

27 Million M, Walter G, Bardin N et al. Immunoglobulin G anticardiolipin antibodies and progression to $Q$ fever endocarditis. Clin Infect Dis 2013;57:57-64.

28 Raoult D, Casalta JP, Richet $\mathrm{H}$ et al. Contribution of systematic serological testing in diagnosis of infective endocarditis. J Clin Microbiol 2005;43:5238-42.

29 Subedi S, Jennings Z, Chen SC. Laboratory Approach to the Diagnosis of Culture-Negative Infective Endocarditis. Heart Lung Circ 2017;26:763-71.

30 Brouqui P, Raoult D. Endocarditis due to rare and fastidious bacteria. Clin Microbiol Rev 2001:14:177-207.

31 Tattevin P, Watt G, Revest M et al. Update on blood culturenegative endocarditis. Med Mal Infect 2015;45:1-8.

32 Baddour LM, Wilson WR, Bayer AS et al. Infective Endocarditis in Adults: Diagnosis, Antimicrobial Therapy, and Management of Complications. Circulation 2015;132:1435-86.

33 Goldenberger D, Kunzli A, Vogt $P$ et al. Molecular diagnosis of bacterial endocarditis by broad-range PCR amplification and direct sequencing. J Clin Microbiol 1997:35:2733-9.
34 Moter A, Musci M, Schmiedel D. Molecular methods for diagnosis of infective endocarditis. Curr Infect Dis Rep 2010;12:244-52.

35 Calabrese F, Carturan E, Thiene G. Cardiac infections: focus on molecular diagnosis. Cardiovasc Pathol 2010;19:171-82.

36 Li JS, Sexton DJ, Mick N et al. Proposed modifications to the Duke criteria for the diagnosis of infective endocarditis. Clin Infect Dis 2000;30:633-8.

37 Anantha Narayanan M, Mahfood Haddad T, Kalil AC et al. Early versus late surgical intervention or medical management for infective endocarditis: a systematic review and meta-analysis. Heart 2016;102:950-7.

38 Chu VH, Park LP, Athan E et al. Association between surgical indications, operative risk, and clinical outcome in infective endocarditis: a prospective study from the International Collaboration on Endocarditis. Circulation 2015;131:131-40.

Address for correspondence: Dr Rachael James, Cardiology Department, Royal Sussex County Hospital, Brighton and Sussex University Hospitals NHS Trust, Brighton BN2 5BE, UK. Email: rachael.james3@nhs.net

\section{Royal College of Physicians}

\section{Rebuilding the NHS RCP priorities for the resetting of services}

The RCP has set out nine key priorities for the resetting and rebuilding of NHS services, now that the initial COVID-19 peak has passed.

The publication covers the role of the medical specialties and emphasises that our healthcare system must not simply return to how it was pre-pandemic. From routine care to reducing health inequalities, particularly for people from ethnic minority backgrounds, we have the opportunity to embed long-term improvements.

Download the document: www.rcplondon.ac.uk/rebuilding-the-NHS



